\title{
High density of CD68+ tumor-associated macrophages predicts a poor prognosis in gastric cancer mediated by IL-6 expression
}

\author{
CHONG-YU SU* ${ }^{*}$ XIAO-LONG FU*, WEI DUAN, PEI-WU YU and YONG-LIANG ZHAO \\ Department of General Surgery and Center of Minimally Invasive Gastrointestinal Surgery, \\ Southwest Hospital, Third Military Medical University, Chongqing 400038, P.R. China
}

Received February 1, 2016; Accepted January 10, 2018

DOI: $10.3892 / \mathrm{ol} .2018 .8119$

\begin{abstract}
The aim of the present study was to explore the potential role of cluster of differentiation CD68+ tumor-associated macrophages (TAMs) induced by interleukin (IL)-6 in the progression of gastric cancer (GC) and patient prognosis. The expression levels of IL-6 and CD68 were detected by immunohistochemical staining in 60 samples of tumor and non-tumor gastric tissues. CD14+ monocytes were isolated from peripheral blood mononuclear cells and stimulated with macrophage colony stimulation factor (M-CSF) and IL-6, and the expression levels of IL-10, IL-12, vascular endothelial growth factor (VEGF)-C and transforming growth factor (TGF)- $\beta$ were measured by reverse transcription polymerase chain reaction and ELISA. The GC MGC-803 cell line was co-cultured with monocytes stimulated by M-CSF and IL-6 and the invasion ability of the MGC-803 was evaluated by Transwell analysis. The levels of STAT3, P-STAT3 and interferon-regulatory factor 4 (IRF4) in the monocytes stimulated by M-CSF and IL- 6 were detected by western blotting. The results demonstrated that the frequencies of IL-6+ macro-

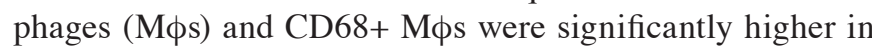
tumor regions compared with the corresponding non-tumor regions of GC tissues. Kaplan-Meier analysis revealed that the densities of tumor-infiltrating CD68+ or IL-6+ Mфs were inversely associated with the overall survival rates of the patients. In vitro, the expression levels of IL-10, VEGF-C and TGF- $\beta$ significantly increased in CD14+ monocytes subsequent to M-CSF and IL-6 stimulation. The invasion abilities of MGC-803 were increased by the monocytes stimulated with M-CSF and IL-6. The levels of STAT3, P-STAT3 and IRF4
\end{abstract}

Correspondence to: Professor Yong-Liang Zhao, Department of General Surgery and Center of Minimally Invasive Gastrointestinal Surgery, Southwest Hospital, Third Military Medical University, 30 Gaotanyan Street, Shapingba, Chongqing 400038, P.R. China E-mail: yongliang1666@163.com

${ }^{*}$ Contributed equally

Key words: interleukin-6, cluster of differentiation 68, tumor associated macrophages, gastric cancer proteins increased in the monocytes stimulated by M-CSF and IL-6. In conclusion, the results from the present study suggest that a high density of CD68+ TAMs predicts a poor prognosis in GC. IL-6 may polarize the M $\phi$ s and promote tumor invasion through the IL-6/STAT3/IRF4 signaling pathway.

\section{Introduction}

Gastric cancer (GC) is the fifth most common type of cancer, and the third leading cause of cancer mortality in the world (1). The 7th edition of the tumor node metastasis (TNM) staging system indicates the importance of the depth of invasion and the number of lymph nodes metastases involved as major prognostic factors (2). Therefore, it is important to understand the mechanisms involved in gastric cancer progression and lymphatic metastasis.

The association between inflammation and cancer was proposed $>100$ years ago, and experimental, clinical and epidemiological studies have revealed that chronic inflammation contributes to cancer progression and may increase predisposition to different types of cancer $(3,4)$. Macrophages (Mфs) are one of the most important inflammatory cells in the tumor microenvironment (4,5). There are two types, M1 and M2, and they are derived from monocytes. M1 macrophages are produced by the classical complement pathway, and are pro-inflammatory. M2 macrophages are activated through the alternative complement pathway, and secrete anti-inflammatory cytokines and participate in tissue repair (6). M1 M $\phi$ s produce large amounts of nitric oxide by expressing inducible nitric oxide synthase (iNOS) and tumor necrosis factor (TNF), and are essential for clearing bacterial, viral and fungal infections (7). M2 M $\phi$ s serve a role in the response to parasite infection, tissue remodeling, angiogenesis and tumor progression (8). In a previous study investigating GC, Ishigami et al (9) suggested that the expression of tumor-associated M $\phi \mathrm{s}$ (TAMs) was positively correlated with stomach lesions, tumor staging and lymph node metastasis. The differentiation of M $\phi \mathrm{s}$ into the M1 or M2 phenotype is affected by the microenvironment, and numerous types of cytokines present. Amongst the tumor-derived factors that modulate myeloid cell polarization, macrophage colony stimulation factor (M-CSF) promotes the recruitment and survival rates of $\mathrm{M} \phi$, and $\mathrm{M}-\mathrm{CSF}$ enhances tumor growth and aggressiveness by stimulating the protumor activities of TAMs (10). 
Interleukin (IL)-6 is a strong activator of signal transducer and activator of transcription 3 (STAT3), and IL-6-dependent STAT3 activation serves a pivotal role in tumor progression. It has been demonstrated in previous studies that IL- 6 is an independent risk factor for poor prognosis inpatients with GC (11). Also, it has been reported that interferon-regulatory factor 4 (IRF4) may regulate $\mathrm{M} 2 \mathrm{M} \phi$ polarization, and contribute to the expression of M2 makers $(8,11)$. In the present study, the significance of the correlation between IL-6 and TAMs, and their role in GC progression and lymph node metastasis was investigated. Furthermore, it was demonstrated that M-CSF and IL-6 drive the differentiation of M2 polarization of TAMs through the IL-6/STAT3/IRF4 signaling pathway, and that in vitro induced M $\phi$ s express high levels of IL-10, transforming growth factor (TGF)- $\beta$ and vascular endothelial growth factor (VEGF)-C. The elevated expression level of STAT3 indicates the activation of the IL-6 signaling pathway. Collectively, these data suggest that the IL-6/STAT3/IRF4 axis may facilitate tumor progression and lymph node metastasis in the GC microenvironment.

\section{Materials and methods}

Clinical data. GC tissue was collected from 60 patients by gastrectomy, subsequently embedded in paraffin, and diagnosed at the Southwest Hospital of Third Military Medical University (Chongqing, China). None of the patients had received radiation or chemotherapy prior to surgery, and clinicopathological classification was confirmed by a pathologist. The age of the patients ranged between 28 to 82 years, and the median age was 54 years. There were 33 males and 27 females. According to the 2010 edition of Union for International Cancer Control staging of GC (12), there were 7 patients with stage I, 14 patients with stage II, 34 patients with stage III and 5 patients with stage IV. According to tumor location, there were 11 patients with gastric cardia, 24 patients with gastric body and 25 patients with antrum. There were 42 patients with lymph node metastasis and 18 patients without lymph node metastasis, as summarized in Table I. The present study was approved by the Ethics Review Board at the Third Military Medical University, and written informed consent was obtained from all patients.

Immunohistochemical staining. The GC tissues were fixed with $10 \%$ paraformaldehyde, and the paraffin-embedded specimens were then cut into $5 \mu \mathrm{m}$ sections. Subsequent to dewaxing and rehydration, the sections were submerged in EDTA buffer and heated in a microwave oven $\left(100^{\circ} \mathrm{C}\right)$ for antigenic retrieval. The sections were the treated with $3 \%$ hydrogen peroxide in methanol to prohibit the endogenous peroxidase activity, and incubated with normal goat serum at $37^{\circ} \mathrm{C}$ for 30 min. Rabbit antibodies against IL-6 (1:400; cat. no. ab6672) and CD68 (1:400; cat. no. Ab125212; Abcam, Cambridge, MA, USA) were incubated with the sections overnight at $4^{\circ} \mathrm{C}$. Subsequent to washing, the tissue sections were treated with horseradish peroxidase labeled anti-rabbit antibody (1:100; cat. no. A0208; Beyotime Institute of Biotechnology, Haimen, China) at $37^{\circ} \mathrm{C}$ for $30 \mathrm{~min}$. The sections were immersed in 3-amino-9-ethyl carbazole (DAB; OriGene Technologies). Subsequent to washing, the tissue sections were counterstained with $10 \%$ hematoxylin, and mounted in Clear-Mount. The sections were then evaluated at magnification, $\mathrm{x} 100$, and 5 representative areas where high densities ( $>5$ cells/field) of IL-6 or CD68 marker (brown) accumulation at were identified. They were then counted at magnification, $x 200$ in each case, and the average value was used. All protocols were performed by 2 researchers (Third Military Medical University) who were blinded to the groups, with assistance from a professional pathologist.

Cell culture. The blood samples were obtained from the Department of Hematology, Southwest Hospital, Chongqing, China. The peripheral blood mononuclear cells (PBMC) were separated by centrifugation at $20^{\circ} \mathrm{C}$ and $2,000 \times \mathrm{g}$ for 20 min with lymphocyte separation medium (GE Healthcare Bio-Sciences, Pittsburgh, PA, USA). CD14+ cells were isolated from the PBMC with the Human CD14 Positive Selection kit (Stemcell Technologies, Inc., Vancouver, Canada). The purified monocytes were then cultured with RPMI-1640 (Gibco; Thermo Fisher Scientific, Inc., Waltham, MA, USA) supplemented with $10 \%$ fetal bovine serum (Hyclone, Logan, UT, USA) and $100 \mathrm{ng} / \mathrm{ml}$ of M-CSF. Fresh media and cytokines were added on day 3. Subsequent to 6 days of induction, the cells were stimulated for $24 \mathrm{~h}$ with or without 50 or $100 \mathrm{ng} / \mathrm{ml}$ human recombinant IL-6 (PeproTech, Inc., Rocky Hill, NJ, USA). All cells were grown at $37^{\circ} \mathrm{C}$ in a humidified atmosphere of $5 \% \mathrm{CO}_{2}$.

Reverse transcription quantitative polymerase chain reaction $(R T-q P C R)$. Total RNA was extracted from cultured cells using TRIzol ${ }^{\circledR}$ reagent according to the manufacturers' protocol (Invitrogen; Thermo Fisher Scientific, Inc., Waltham, MA, USA). The RNA (1,000 ng in $10 \mu \mathrm{l}$ volume) was reverse transcribed with a reverse transcription kit (Takara Bio., Inc., Otsu, Japan) at $37^{\circ} \mathrm{C}$ for $15 \mathrm{~min}$, then $85^{\circ} \mathrm{C}$ for $12 \mathrm{sec}$. cDNA was obtained and diluted with $20 \mu \mathrm{l}$ nuclease free water. qPCR was performed on a BIO-RAD CFX96-Tm Real-Time System (Bio-Rad Laboratories, Inc., Hercules, CA, USA) by mixing $2 \mu 1$ diluted cDNA with SYBR Green Master Mix (Applied Biosystems, MA, USA). The reaction conditions were as follows: $95^{\circ} \mathrm{C}$ for $2 \mathrm{~min}, 40$ cycles at $95^{\circ} \mathrm{C}$ for $5 \mathrm{sec}, 55^{\circ} \mathrm{C}$ for $30 \mathrm{sec}$, and $60^{\circ} \mathrm{C}$ for $2 \mathrm{~min}$. The forward and reverse primers (Shanghai GenePharma Co., Ltd., Shanghai, China) listed in Table II. The relative expression levels of the target mRNAs (IL-10, IL-12, VEGF-C and TGF- $\beta$ ) were using the $2^{-\Delta \Delta C q}$ method, using $\beta$-actin as a calibrator (13).

ELISA analysis. The cytokines IL-10, IL-12, TGF $\beta$ and VEGF-C in the supernatants of the cultured monocytes, centrifuged at $500 \mathrm{x} \mathrm{g}$ for $5 \mathrm{~min}$ at $20^{\circ} \mathrm{C}$, were examined using an ELISA, following the protocol of the manufacturer (BioLegend, Inc., San Diego, CA, USA). All of the samples were measured in triplicate.

Transwell analysis. Subsequent to 6 days of differentiation of the monocytes with M-CSF, IL-6 was added to a final concentration of $100 \mathrm{ng} / \mathrm{ml}$ and they were cultured for an additional 24 h. Subsequently, $1 \times 10^{5}$ MGC-803 cells were seeded in the upper well of the Transwell in RPMI 1640 with 5\% fetal calf serum (FCS) and $1 \times 10^{5} \mathrm{M} \phi$ s or IL- 6 induced M $\phi$ s were 
Table I. Association of IL-6+ cells and CD68+ cells with clinicopathological characteristics.

\begin{tabular}{|c|c|c|c|c|c|c|}
\hline \multirow[b]{2}{*}{ Clinical parameter } & \multicolumn{3}{|c|}{ CD68 expression } & \multicolumn{3}{|c|}{ IL-6 expression } \\
\hline & Low, n & High, $n$ & P-value & Low, $\mathrm{n}$ & High, $\mathrm{n}$ & P-value \\
\hline \multicolumn{7}{|l|}{ Age, years } \\
\hline$<54$ & 10 & 16 & 0.181 & 14 & 12 & 0.228 \\
\hline$\geq 54$ & 19 & 15 & & 13 & 21 & \\
\hline \multicolumn{7}{|l|}{ Sex } \\
\hline Male & 18 & 15 & 0.287 & 16 & 17 & 0.586 \\
\hline Female & 11 & 16 & & 15 & 12 & \\
\hline \multicolumn{7}{|l|}{ Location } \\
\hline Cardia & 4 & 7 & 0.408 & 6 & 5 & 0.607 \\
\hline Body & 14 & 10 & & 11 & 13 & \\
\hline Sinuses & 11 & 14 & & 15 & 10 & \\
\hline \multicolumn{7}{|l|}{ TNM Stage } \\
\hline $\mathrm{I}+\mathrm{II}$ & 16 & 5 & 0.007 & 14 & 7 & 0.004 \\
\hline III+IV & 14 & 25 & & 11 & 28 & \\
\hline \multicolumn{7}{|c|}{ Lymph node metastasis } \\
\hline Yes & 12 & 30 & 0.018 & 14 & 28 & 0.017 \\
\hline No & 11 & 7 & & 12 & 6 & \\
\hline
\end{tabular}

TNM, tumor node metastasis.

Table II. Primers for quantitative expression of IL-10, IL-12, VEGF-C and TGF- $\beta$.

\begin{tabular}{lllr}
\hline Cytokine & \multicolumn{1}{c}{ Forward } & Reverse & Length, bp \\
\hline IL-10 & 5'-GCTGTCATCGATTTCTTCCC-3' & 5'-CTCATGGCTTTGTAGATGCCT-3' & 103 \\
IL-12 & 5'-AGGGCCGTCAGCAACATG-3' & 5'-TCTTCAGAAGTGCAAGGGTAAAATTC-3' & 68 \\
VEGF-C & 5'-CAGCACGAGCTACCTCAGCAAG-3' & 5'-TTTAGACATGCATCGGCAGGAA-3' & 117 \\
TGF- $\beta$ & 5'-AACTACTGCTTCAGCTCCAC-3' & 5'-TGTGTCCAGGCTCCAAATGTA-3' & 155 \\
\hline
\end{tabular}

IL, interleukin; VEGF-C, vascular endothelial growth factor C; TGF- $\beta$, transforming growth factor $\beta$.

seeded on the lower surface of the inverted filter membrane in RPMI 1640 with 5\% FCS. Subsequent to 1 day of incubation at $37^{\circ} \mathrm{C}$, the cells fixed with $3.7 \%$ paraformaldehyde in CS buffer, $0.1 \mathrm{M}$ piperazine-N,N'-bis (2-ethanesulfonic acid; PIPES), 1 mM EGTA, 4\% polyethylene glycol 800 and $0.1 \mathrm{M} \mathrm{NaOH}$, for $20 \mathrm{~min}$ at room temperature for subsequent crystal violet staining. The number of MGC-803 cells that penetrated the micropores were then counted at magnification x 200 with a light-microscope (Nikon Corporation, Tokyo, Japan) (14).

Western blot analysis. Total protein was extracted from the cells cultured for 7 days with or without IL-6 stimulation, and the concentration of proteins was determined using a BCA protein assay kit (Nanjing KeyGen Biotech., Co., Ltd., Nanjing, China). Equal quantities of protein were separated by $10 \%$ SDS-PAGE gels and transferred onto polyvinylidene difluoride membranes (EMD Millipore, Billerica, MA, USA). Subsequent to blocking for $1 \mathrm{~h}$ with $3 \%$ bovine serum albumin, the membranes were incubated overnight at $4^{\circ} \mathrm{C}$ with primary antibody against STAT3 (dilution, 1:1,000; cat. no. ab68153), P-STAT3 (dilution, 1:1,000; cat. no. ab76315), IRF4 (dilution, 1:1,000; cat. no. ab133590; all Abcam), and $\beta$-actin (dilution, 1:2,000; cat. no. sc-130656; Santa Cruz Biotechnology, Inc., Dallas, TX, USA). Subsequently, the membranes were washed with $0.1 \%$ TBS-Tween-20 and incubated with goat anti-mouse or rabbit horseradish peroxidase-conjugated secondary antibodies (dilution, 1:5,000; Jackson ImmunoResearch Laboratories, Inc., West Grove, PA, USA) for $1 \mathrm{~h}$ at room temperature. The blots were detected using the SuperSignal ${ }^{\mathrm{TM}}$ West Dura Extended Duration Substrate enhanced chemiluminescence western blotting kit (Pierce; Thermo Fisher Scientific, Inc.).

Statistical analysis. The statistical analysis between the groups was performed by the Mann-Whitney U test. Overall survival (OS) rates of patients and their differences were determined by Kaplan-Meier method and log-rank test. 
$\mathbf{A}$
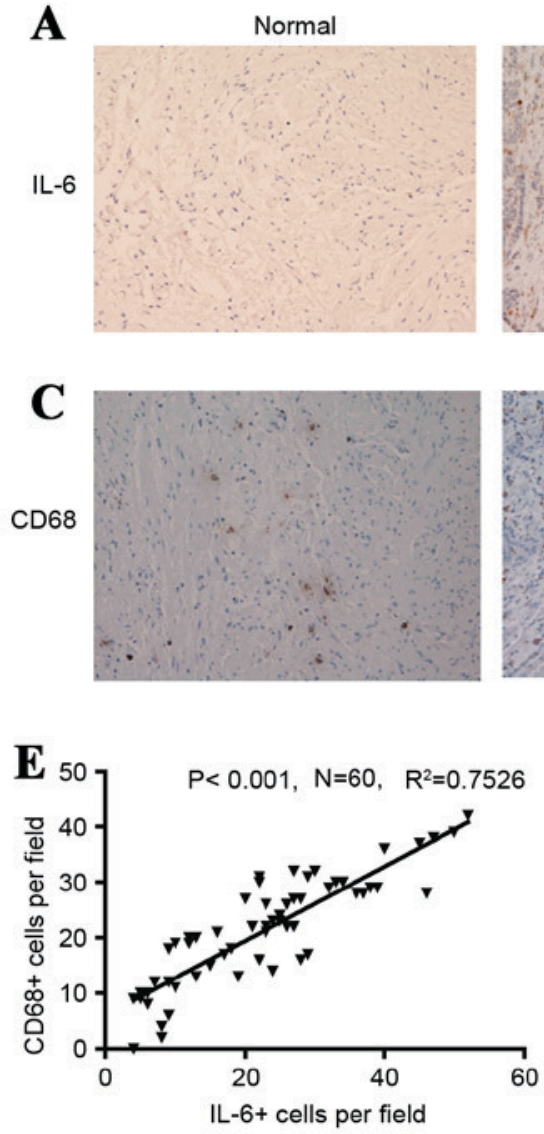
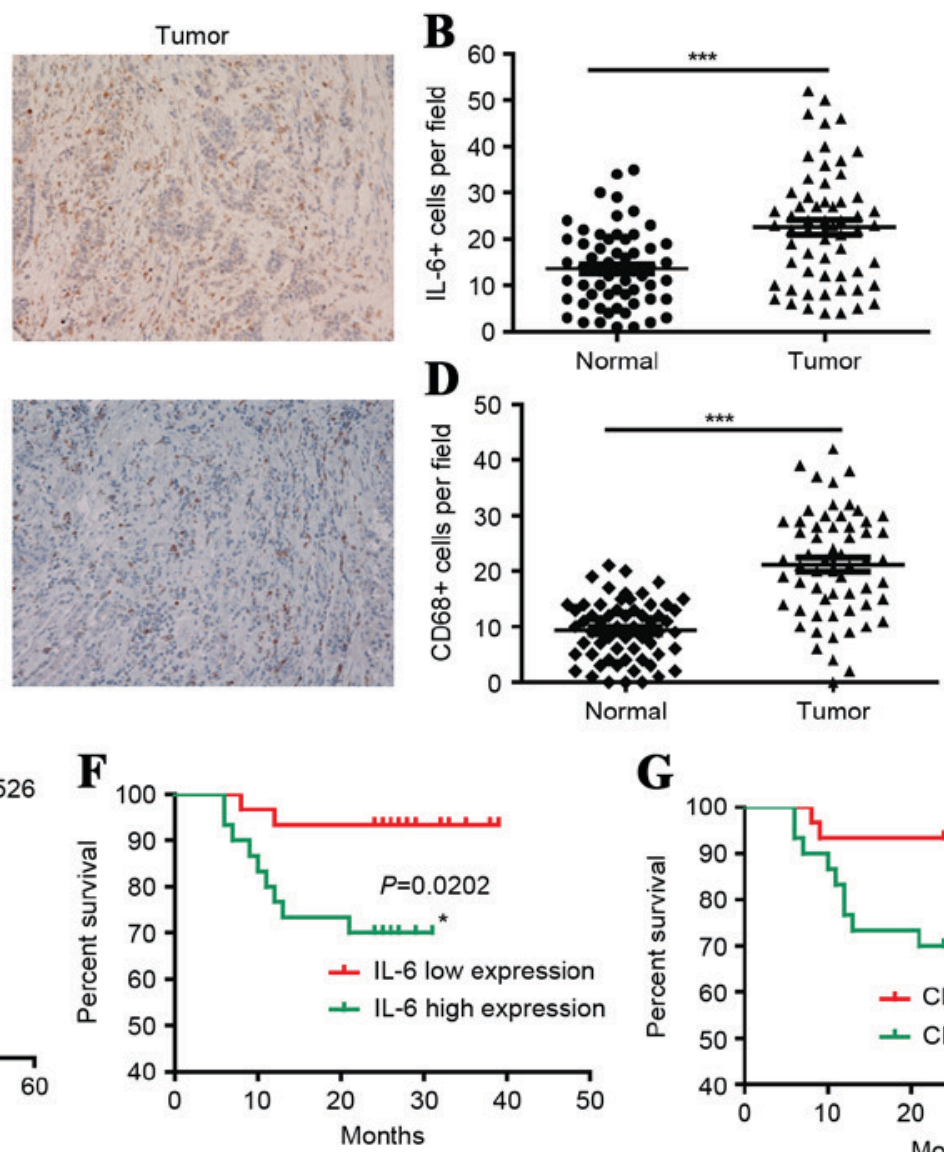

G

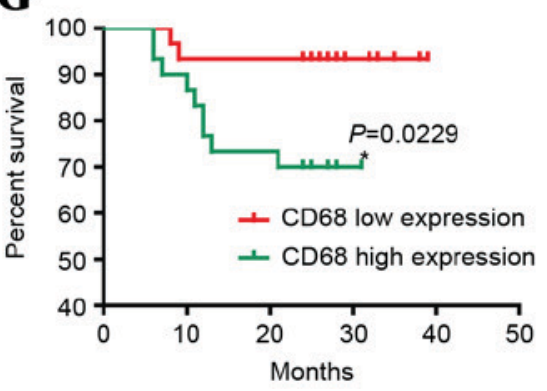

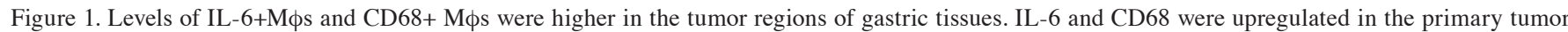
tissues and correlated with poor prognosis of patients with gastric cancer. (A) Representative immunohistochemistry images of IL-6+M $\phi s$ (magnification, x100). (B) Numbers of IL- $6+\mathrm{M} \phi \mathrm{s}$ in 60 pairs of matching tumor and normal tissues. (C) Representative immunohistochemistry images of CD68+M $\phi s$ (magnification, x100). (D) Numbers of CD68+M $\phi s$ in 60 pairs of matching tumor and normal tissues. (E) Scatter demonstrates that the positive correlation between the expression levels of IL- 6 and CD68 in the 60 gastric tumor tissues $\left(\mathrm{R}^{2}=0.7526\right.$; $\left.\mathrm{P}<0.01\right)$. (F) Kaplan-Meier survival estimates and log-rank tests were used to analyze the prognostic significance of IL-6+M $\phi s$ and (G) CD68+M $\phi$ s in the tumor regions of gastric tissues. Patients were divided into 2 groups according to the median number of $\mathrm{M} \phi$ s per $\mathrm{x} 400$ field. ${ }^{* * *} \mathrm{P}<0.01$. Red lines, low group; green lines, high group; M $\phi s$, macrophages; IL, interleukin; CD, cluster of differentiation.

Analyses were performed using GraphPad Prism software, version 5.0 (GraphPad Software, Inc., La Jolla, CA, USA). $\mathrm{P}<0.05$ was considered to indicate a statistically significant difference. All of the data are presented as the mean \pm standard deviation.

\section{Results}

The level of IL-6+ and CD68+M regions of gastric tissues. M $\phi s$ exhibit diverse phenotypes during inflammation and tumor pathogenesis. To evaluate the types of $\mathrm{M} \phi$ phenotype and distribution patterns in human GC tissues, the present study used immunostaining to investigate IL-6+ and CD68+ M $\phi$ s in situ. IL-6+ M $\phi$ s were revealed to be distributed throughout the tissues, and were more frequent in the tumor regions compared with non-tumor regions of gastric tissues, $23 \pm 13$ and $13 \pm 8$ cells/field, respectively, as demonstrated in Fig. 1. It was also demonstrated that CD68+ $\mathrm{M} \phi \mathrm{s}$ were distributed throughout the tissues, and were more frequent in tumor regions than non-tumor regions of gastric tissues, $21 \pm 10$ and $9 \pm 5$ cells/field, respectively, as illustrated in Fig. 1. Additionally, the frequencies of IL-6+ cells were positively associated with the frequencies of CD68+ cells. These results indicate that the level of IL-6+M $\phi$ s and CD68+ $\mathrm{M} \phi \mathrm{s}$ is higher in the tumor regions of gastric tissues.

IL-6 and CD68 were correlated with poor clinical pathological features and prognosis of GC patients. As demonstrated in Table I, the expression levels of IL-6 and CD68 were positively associated with lymph node metastasis and TNM stages. The difference was statistically significant $(\mathrm{P}<0.05)$. In addition, to investigate the associations between IL- 6 and CD68 with GC progression, 60 patients with GC were divided into 2 groups based on the median frequencies of IL-6+ and CD68+ Mфs, respectively. Kaplan-Meier survival curves were then plotted to investigate the association with OS rate, as demonstrated in Fig. 1. The log-rank statistic was used to compare OS rates between the low and high IL-6 or CD68 expression groups. There was a markedly positive association between OS and the densities of IL-6+ $(\mathrm{P}=0.0202)$ and CD68+ $\mathrm{M} \phi \mathrm{s}(\mathrm{P}=0.0229)$, as illustrated in Fig. $1 \mathrm{~F}$ and $\mathrm{G}$, respectively. These results indicate that IL- 6 and CD68 exhibit a positive correlation with poor clinical pathological features and prognosis of patients with GC. 

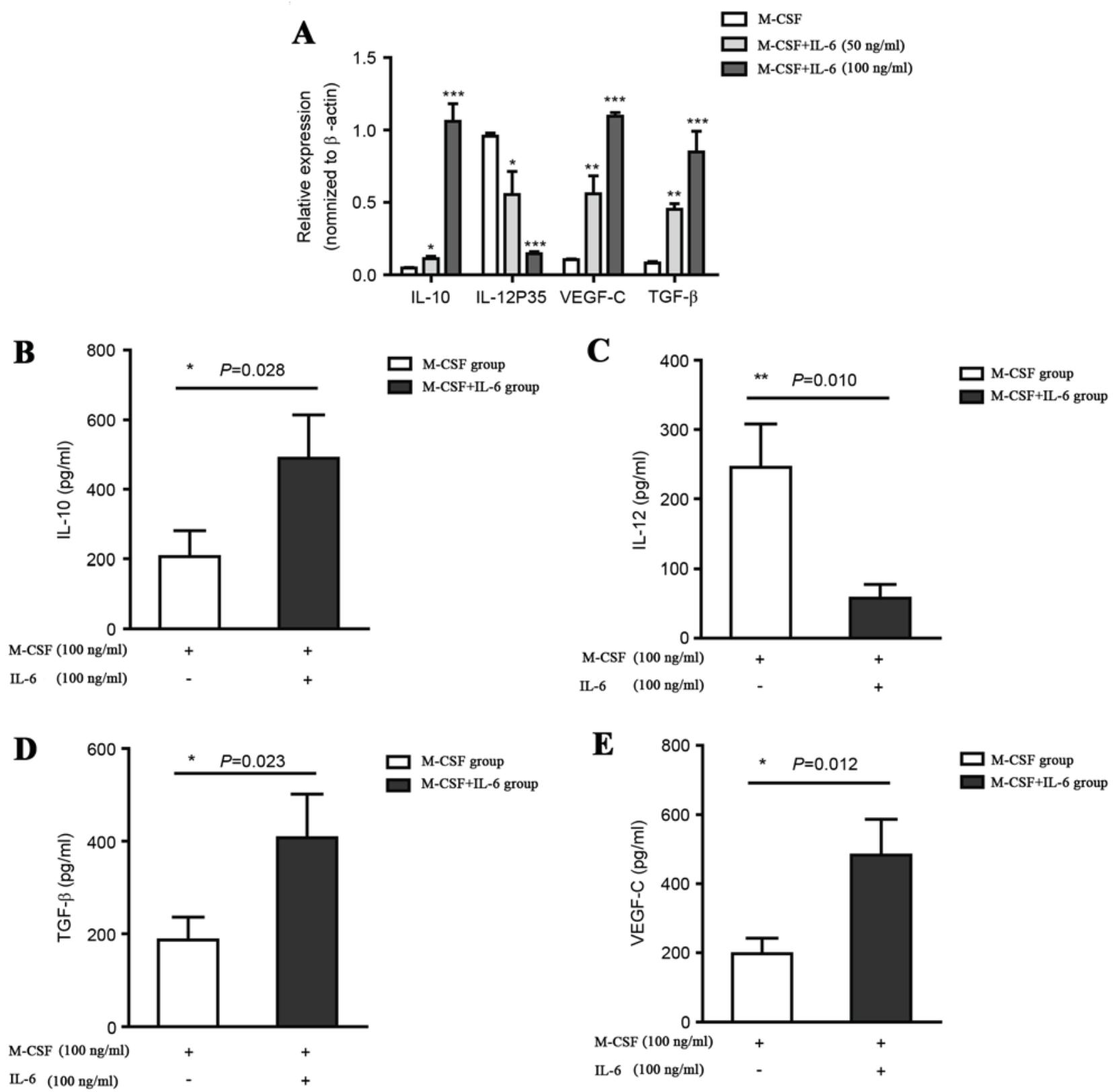

Figure 2. IL-10, VEGF-C and TGF- $\beta$ levels increase and IL-12 levels decrease in CD14+ monocytes and supernatants of cultured monocytes stimulated with M-CSF alone, or M-CSF and IL-6. (A) Relative levels of IL-10, IL-12, VEGF-C and TGF- $\beta$ in CD14+ monocytes stimulated with M-CSF alone, or M-CSF and IL- 6 at concentration of 50 and $100 \mathrm{ng} / \mathrm{ml}$, quantified by reverse transcription quantitative polymerase chain reaction. Data are presented as the mean \pm standard deviation ( $\mathrm{n}=6, \mathrm{n}$, number of samples). (B) Measurements of IL-10 (C) IL-12 (D) VEGF-C and (E) TGF- $\beta$ in supernatants of cultured monocytes stimulated with M-CSF alone, or M-CSF and $100 \mathrm{ng} / \mathrm{ml}$ IL- 6 were performed using ELISA in triplicate. ${ }^{*} \mathrm{P}<0.05$, ${ }^{* * *} \mathrm{P}<0.01,{ }^{* * * *} \mathrm{P}<0.001 \mathrm{vs}$. M-CSF. M-CSF, macrophage colony stimulation factor; IL, interleukin; TGF- $\beta$, transforming growth factor $\beta$; VEGF-C, vascular endothelial growth factor C.

Expression levels of IL-10,IL-12,VEGF-C and TGF- $\beta$ in the CD14+ monocytes and in supernatants of cultured monocytes stimulated with M-CSF alone, or M-CSF and $I L-6$. As M-CSF may induce monocyte differentiation into $\mathrm{M} \phi \mathrm{s}$, it has been demonstrated that $\mathrm{M} 1 \mathrm{M} \phi$ s produce pro-inflammatory cytokines such as IL-12 and exert an antitumor effect, whilst M2 M $\phi$ s produce IL-10, VEGF-C and TGF- $\beta$ and promote tumor progression. Therefore, CD14+ monocytes were cultured for 6 days with $100 \mathrm{ng} / \mathrm{ml}$ M-CSF and stimulated for $24 \mathrm{~h}$ with or without $100 \mathrm{ng} / \mathrm{ml}$ IL-6. The M $\phi$ s induced by IL- 6 exhibited an M2 polarized phenotype, IL-10 $0^{\text {high }}, \mathrm{IL}-12^{\text {low }}$, and high expression levels of lymph angiogenesis-promoting 1 factor VEGF-C and tumor progression-promoting factor TGF- $\beta$ compared with the control group, which was cultured with M-CSF, as demonstrated in Fig. 2A. In addition, the differentiation of M $\phi$ s was also detected with ELISA analysis. CD14+ monocytes from the IL-6-treated model exhibited higher IL-10 and TGF- $\beta$ expression levels but lower IL-12 level compared with the control group, as illustrated in Fig. 2, which supports the hypothesis that IL- 6 treatment promotes the differentiation of M2 M $\phi s$. The IL-6-treated M2 M $\phi s$ also generated a high expression level of VEGF-C, which supported the hypothesis of the ability of M2 M $\phi$ s to induce the formation of lymphatic fluid. To determine the effect of TAMs on VEGF/VEGF-C production, the expression levels of VEGF and VEGF-C in 

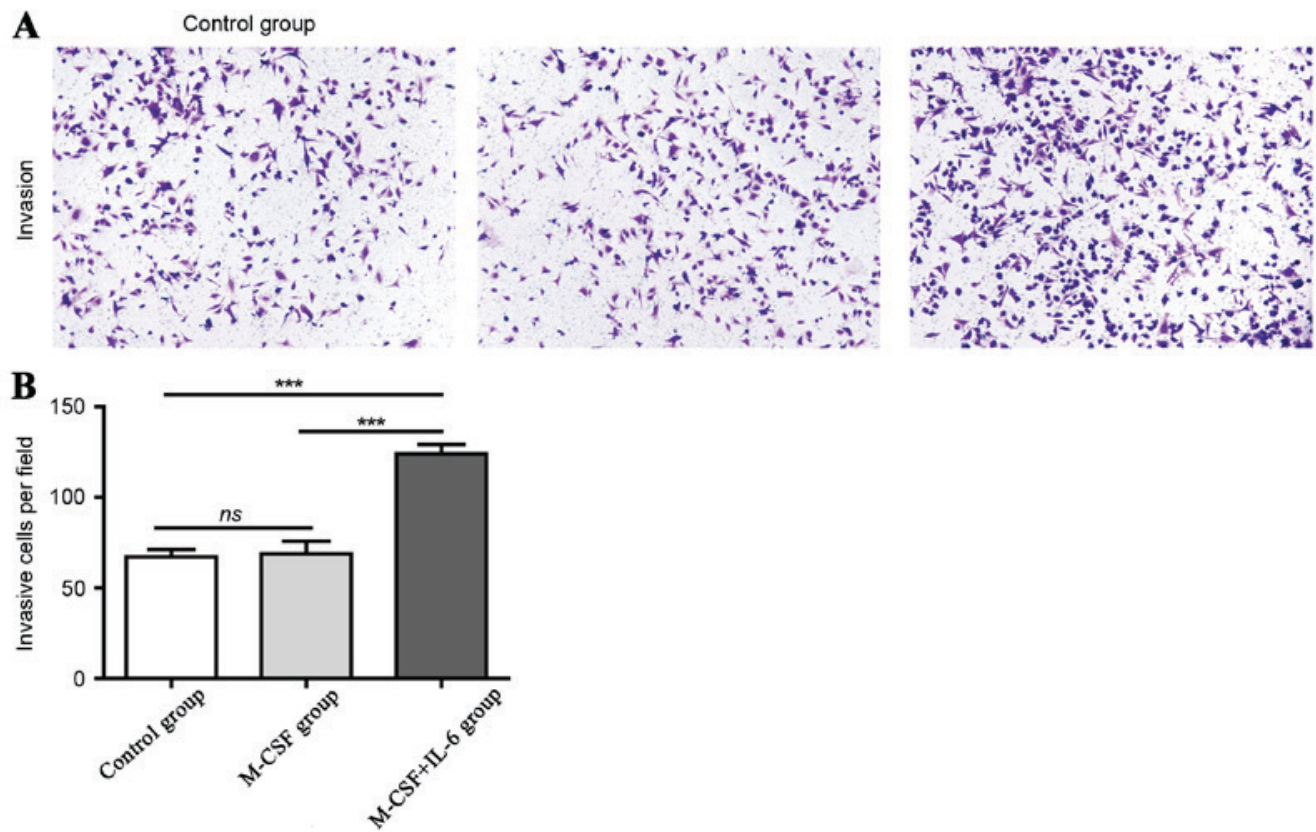

Figure 3. Invasion abilities of MGC803 were increased by monocytes stimulated with M-CSF and IL-6. (A) Representative images Crystal Violet Staining at magnification, $x 200$ depicting the invasion ability of MGC-803 subsequent to co-culturing with macrophages stimulated with M-CSF alone, or M-CSF and IL-6. (B) Data are presented as mean \pm standard deviation. ( $\mathrm{n}=5$, n, number of samples) and analyzed by a two-tailed unpaired $\mathrm{t}$-test $\left({ }^{* * * *} \mathrm{P}<0.001\right)$, NS, not significant. M-CSF, macrophage colony stimulation factor; IL, interleukin.

M $\phi$ s induced and not induced by IL- 6 were examined. In the IL-6-inducted model, a significant increase in the expression of VEGF and VEGF-C in the M $\phi$ s was detected, indicating that the TAMs were a source of VEGF and VEGF-C in the tumor microenvironment.

TAMs from IL-6-treated M and metastasis capacity of tumor cells. It has been suggested that TAMs are critical regulators of the tumor microenvironment and directly affect the growth, survival, invasion and metastasis of tumor cells. In the Transwell assay analysis, it was identified that there were $\sim 70$ cells/field in the control and the Mфs-elicited groups. In contrast, there were $\sim 130$ cells per field in the IL- 6 induced M2 M $\phi$ s group, which supports the hypothesis that TAMs may promote the invasion of tumor cells, as demonstrated in Fig. 3A and B.

Janus kinase/signal transducers and activators of transcription 3 (JAK/STAT3) signal pathway was activated by $I L-6$ and resulted in the activation of IRF4. IL-6 is a major cancer-promoting cytokine, which induces several pathways that lead to tumor growth, survival, angiogenesis, and drug resistance. In the present study, it was identified that IL-6 induced $\mathrm{M} \phi$ differentiation into the M2 phenotype, but the mechanism of this method of differentiation remains unknown. To additionally explore the mechanism of IL-6 inducing $\mathrm{M} \phi$ s polarized in the in vitro culture system, the present study used western blot analysis to detect the production of IRF4, STAT3 and P-STAT3. The results from the western blot analysis demonstrated that the levels of expression of IRF4, STAT3 and P-STAT3 proteins in M2 M $\phi$ cells was markedly elevated compared with non-IL-6-induced $\mathrm{M} \phi$ s, as illustrated in Fig. 4.

\section{M-CSF+IL-6 M-CSF}

STAT3
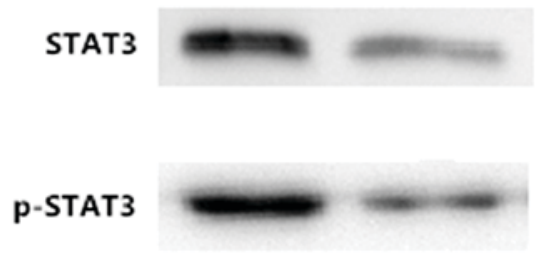

IRF4

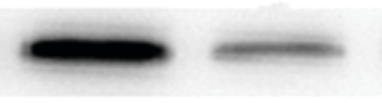

$\beta$-actin

Figure 4. Levels of STAT3, P-STAT3 and IRF4 proteins were increased in the monocytes stimulated by M-CSF and IL-6. Levels of STAT3, P-STAT3 and IRF4 protein in the M-CSF group and M-CSF+IL-6 group were detected by western blotting. $\beta$-actin was used as the loading control. The assays were repeated in duplicate. STAT3, signal transducer and activator of transcription 3; pSTAT3, phosphorylated STAT3; M-CSF, macrophage colony stimulation factor; IL, interleukin, IRF4, interferon-regulatory factor 4 .

\section{Discussion}

$G C$ is one of the most common types of malignant tumor in the digestive tract, with high rates of incidence and mortality. The global incidence rate is 952,000 cases of GC and 723,000 incidences of mortality in $2012(1,15,16)$. The formation of lymphatic vessels and subsequent lymphatic metastasis is the primary cause of mortality in patients with GC (17). Therefore, 
studies investigating the mechanisms of lymphangiogenesis may identify novel methods for improving the effects of treatment and prognosis of GC. The present study investigated IL-6 and CD68+ M $\phi$ s in the GC microenvironment, and the correlation between prognosis. In addition, aspects of the IL-6-dependent induction of CD14+ monocytes M $\phi$ differentiation into M2-like M $\phi$ s in vitro have been revealed.

The tumor microenvironment consists of tumor cells, mesenchymal cells and the infiltrated immune cells, and supports tumor growth and progression. It has been demonstrated that the infiltration of inflammatory immune cells into the tumor microenvironment is important in the mechanisms of tumor progression, invasion and metastasis. Previous studies have identified TAMs as a type of inflammatory immune cell present in tumor tissues, and that the number of TAMs is positively correlated with tumor lymphatic metastasis and poor prognosis (18-20). Analysis of immunohistochemical staining of GC and normal tissues has revealed that the expression levels of VEGF-C and IL-6 are positively correlated with the levels of CD68+ M $\phi s$ in $\mathrm{GC}$ tissues, that the expression of VEGF-C in GC tissues is significantly higher compared with normal tissues and the level of VEGF-C is positively correlated with the density of lymphatic vessels, levels of lymph node metastasis of GC tissue and poor prognosis of patients.

IL-6 is a major cancer-promoting cytokine, which induces several pathways that lead to tumor growth, survival, angiogenesis and drug resistance. The immunohistochemical staining results of the present study demonstrate that the level of IL- 6 increased in the tumor microenvironment, which induces $\mathrm{M} \phi$ differentiation into TAMs, consequently promoting tumor progression. The present study also demonstrated that the expression levels of IL-6, CD68+ $\mathrm{M} \phi \mathrm{s}$ and VEGF-C are positively correlated with the number of new lymphatic vessels, the depth of GC invasion, lymph node metastasis and TNM stages. The results of the immunohistochemical double-staining analysis indicated that IL-6 is expressed in TAMs, whilst a previous study (21) has demonstrated that there is an IL- 6 receptor expressed on the surface of TAMs. The present study speculates that IL-6 may induce the differentiation of M $\phi$ s to the M2-like phenotype with high expression levels of IL-10, TGF- $\beta$ and VEGF-c, and low expression levels IL-12 through paracrine signaling.

It was revealed that the M $\phi$ s induced by IL- 6 exhibited an M2-like polarized phenotype (IL-10 $0^{\text {high }}$, IL-12 ${ }^{\text {low }}$ ) and high expression levels of lymphangiogenesis-promoting factor VEGF-C and tumor progression-promoting factor TGF- $\beta$ compared with the control group, which was cultured with M-CSF. The supernatants were collected to detect expression levels of IL-10, IL-12, TGF- $\beta$ and VEGF-C. The results suggests a M2 phenotype, which indicates that IL-6 treatment promoted the differentiation of M2-like M $\phi$ s. Subsequently, a Transwell experiment was used to determine if TAMs from IL-6-treated M $\phi$ s exhibited a similar capacity. Subsequent to crystal violet staining, there were $\sim 130$ cells/field in the IL-6 induced M2-like M $\phi s$ group, but $\sim 70$ cells/field in the control group, which supported the hypothesis that IL-6 induced M2-like M $\phi s$ may promote the invasion of tumor cells.

A previous study identified that the activation of the JAK/STAT3 signaling pathway as one of the carcinogenic mechanisms of IL-6 (22), and that transcription factor IRF4 is an important molecule that controls M2 M $\phi$ polarization (8). To examine the mechanism of IL- 6 induction of $\mathrm{M} \phi$ polarization in vitro, western blot analyses were used to detect the production of IRF4, STAT3 and P-STAT3. The results demonstrated that the levels of IRF4, STAT3 and P-STAT3 proteins in IL-6 induced M $\phi$ s was markedly elevated compared with non-IL-6-induced M $\phi$ s, which indicates that IL-6 may elicit the phosphorylation of STAT3 and the activation of the STAT3 signaling pathway, promote the transcription of IRF4 and regulate the production of IL-10 or IL-12, resulting in the M2-like phenotype.

\section{Acknowledgements}

The present study would like to thank the Department of Pathology, Southwest Hospital, Third Military Medical University, Chongquing, China, for their technical assistance.

\section{Funding}

The present study was supported by National Natural Science Foundation of China (grant no. 81372560).

\section{Availability of data and materials}

The datasets used and/or analyzed during the current study are available from the corresponding author on reasonable request.

\section{Author's contributions}

YLZ and PWY designed the experiment, CYS and XLF performed the experiment, WD processed the data and CYS wrote the manuscript. All authors read and approved the final manuscript.

\section{Ethics approval and consent to participate}

The present study was approved by the Ethics Review Board at The Third Military Medical University, and written informed consent was obtained from all patients.

\section{Consent for publication}

Patient, parent or next of kin (in the case of deceased patients) provided written informed consent for the publication of any associated data and accompanying images.

\section{Competing interests}

The authors declare they they have no competing interests.

\section{References}

1. Siegel R, Naishadham D and Jemal A: Cancer Statistics, 2012. CA Cancer J Clin 62: 10-29, 2012.

2. Biondi A and Hyung WJ: Seventh edition of TNM classification for gastric cancer. J Clin Oncol 29: 4338-4342, 2011.

3. Anestakis D, Petanidis S, Kalyvas S, Nday CM, Tsave O, Kioseoglou E and Salifoglou A: Mechanisms and applications of interleukins in cancer immunotherapy. Int J Mol Sci 16: 1691-1710, 2015. 
4. Allavena P, Sica A, Solinas G, Porta C and Mantovani A: The inflammatory micro-environment in tumor progression: The role of tumor-associated macrophages. Crit Rev Oncol Hematol 66: $1-9,2008$.

5. Zhang B, Zhang Y, Yao G, Gao J, Yang B, Zhao Y, Rao Z and Gao J: M2-polarized macrophages promote metastatic behavior of Lewis lung carcinoma cells by inducing vascular endothelia growth factor-C expression. Clinics(Sao Poulo) 67: 901-906, 2012.

6. Mantovani A, Sozzani S, Locati M, Allavena P and Sica A: Macrophage polarization: Tumor-associated macrophages as a paradigm for polarized M2 mononuclear phagocytes. Trends Immunol 23: 549-555, 2002.

7. Benoit M, Desnues B and Mege JL: Macrophage polarization in bacterial infections. J Immunol 181: 3733-3739, 2008.

8. Satoh T, Takeuchi O, Vandenbon A, Yasuda K, Tanaka Y, Kumagai Y, Miyake T, Matsushita K, Okazaki T, Saitoh T, et al The Jmjd3-Irf4 axis regulates M2 macrophage polarization and host responses against helminth infection. Nat Immunol 11: 936-989, 2010.

9. Ishigami S, Natsugoe S, Tokuda K, Nakajo A, Okumura H, Matsumoto M, Miyazono F, Hokita S and Aikou T: Tumor-associated macrophage (TAM) infiltration in gastric cancer. Anticancer Res 23: 4079-4083, 2003.

10. Dominguez-Soto A, Sierra-Filardi E, Puig-Kroeger A, Pérez-Maceda B, Gomez-Aguado F, Corcuera MT, Sánchez-Mateos P and Corbí AL: Dendritic cell-specific ICAM-3-grabbing nonintegrin expression on M2-polarized and tumor-associated macrophages is macrophage-CSF dependent and enhanced by tumor-derived IL-6 and IL-10. J Immunol 186: 2192-2200, 2011.

11. Taniguchi $\mathrm{K}$ and Karin M: IL-6 and related cytokines as the critical lynchpins between inflammation and cancer. Semin Immunol 26: 54-74, 2014

12. Edge SB, Byrd DB, Compton CC, Fritz A, Balch CM, Haller DG, et al: AJCC cancer staging manual, 7th ed. New York Springer-Verlag P117-P126, 2009.

13. Livak KJ and Schmittgen TD: Analysis of relative gene expression data using real-time quantitative PCR and the 2(-Delta Delta C(T)) method. Methods 25: 402-408, 2001.
14. Kasper JY, Hermanns MI, Unger RE and Kirkpatrick CJ: A responsive human triple-culture model of the air-blood barrier: Incorporation of different macrophage phenotypes. J Tissue Eng Regen Med 11: 1285-1297, 2017.

15. Chen W, Zheng R, Baade PD, Zhang S, Zeng H, Bray F, Jemal A, $\mathrm{Yu}$ XQ and He J: Cancer statistics in China, 2015. CA Cancer J Clin 66: 115-132, 2016.

16. Milne AN, Carneiro F, O'Morain C and Offerhaus GJA: Nature meets nurture: Molecular genetics of gastric cancer. Hum Genet 126: 615-628, 2009.

17. Yu JW, Wu JG, Tajima Y, Li XQ, Du GY, Zheng LH, Zhang B, Ni XC and Jiang BJ: Study on lymph node metastasis correlated to lymphangiogenesis, lymphatic vessel invasion and lymph node micrometastasis in gastric cancer. J Surg Res 168: 188-196, 2011

18. Fukuda K, Kobayashi A and Watabe K: The role of tumor-associated macrophage in tumor progression. Front Biosci (Schol Ed) 4: 787-798, 2012.

19. Kurahara H, Takao S, Maemura K, Mataki Y, Kuwahata T, Maeda K, Sakoda M, Iino S, Ishigami S, Ueno S, et al: M2-polarized tumor-associated macrophage infiltration of regional lymph nodes is associated with nodal lymphangiogenesis and occult nodal involvement in $\mathrm{pNO}$ pancreatic cancer. Pancreas 42: 155-159, 2013

20. Zhang BC, Gao J, Wang J, Rao ZG, Wang BC and Gao JF: Tumor-associated macrophages infiltration is associated with peritumoral lymphangiogenesis and poor prognosis in lung adenocarcinoma. Med Oncol 28: 1447-1452, 2011.

21. Cao RH, Björndahl MA, Religa P, Clasper S, Garvin S, Galter D, Meister B, Ikomi F, Tritsaris K, Dissing S, et al: PDGF-BB induces intratumoral lymphangiogenesis and promotes lymphatic metastasis. Cancer Cell 6: 333-345, 2004.

22. Tacke RS, Tosello-Trampont A, Nguyen V, Mullins DW and Hahn YS: Extracellular hepatitis c virus core protein activates STAT3 in human monocytes/macrophages/dendritic cells via an IL-6 autocrine pathway. J Biol Chem 286: 10847-10855, 2011.

(i) (2) This work is licensed under a Creative Commons

c) Attribution-NonCommercial-NoDerivatives 4.0 International (CC BY-NC-ND 4.0) License. 